\title{
Regulation of 2D DNA nanostructures by the coupling of intrinsic tile curvature and arm twist ${ }^{\dagger}$
}

${ }^{\dagger}$ This paper is dedicated to the memory of Professor Nadrian C. Seeman.

Chuan Jiang ${ }^{1, \circ}$, Biao Lu ${ }^{1, \circ}$, Wei Zhang ${ }^{1, \circ}$, Yoel P. Ohayon ${ }^{2}$, Feiyang Feng ${ }^{1}$, Shijie Li ${ }^{1}$, Nadrian C. Seeman $^{2, *}$, Shou-Jun Xiao ${ }^{1, *}$

${ }^{1}$ State Key Laboratory of Coordination Chemistry, School of Chemistry and Chemical Engineering, Nanjing University, Nanjing 210023, China.

${ }^{2}$ Department of Chemistry, New York University, New York, NY 10003, USA

* Corresponding authors. E-mails: ned.seeman@nyu.edu, sjxiao@nju.edu.cn

${ }^{\dagger}$ Prof. Nadrian C. Seeman passed away prior to the submission of this paper for publication.

ABSTRACT: The overwinding and underwinding of duplex segments between junctions have been used in designing both lefthanded and right-handed DNA origami nanostructures. For a variety of DNA tubes obtained from self-assembled tiles, only a theoretical approach of the intrinsic curvature of the DNA tile (specified as the intrinsic tile curvature) has been previously used to explain their formation. Details regarding the quantitative and structural descriptions of the tile curvature and its evolution in DNA tubes by the coupling of the twist of the inter-tile arm (specified as the arm twist) have never been addressed. In this work, we designed three types of tile cores built around a circular 128 nucleotide scaffold by using longitudinal weaving (LW), bridged longitudinal weaving (bLW) and transverse weaving (TW). Joining the tiles with inter-tile arms having the length of an odd number of DNA half-turns (termed O-tiling) almost resulted into planar 2D lattices, whereas joining the tiles with the arms having the length of an even number of DNA half-turns (termed E-tiling) nearly generated tubes. Streptavidin bound to biotin was used as a labeling technique to characterize the inside and outside surfaces of the E-tiling tubes and thereby the conformations of their component tiles with addressable concave and convex curvatures. When the arms have the normal winding at the relaxed B-form of DNA, the intrinsic tile curvature determines the chirality of the E-tiling tubes. By regulating the arm length and the sticky end length of the bLW-Ep/q (E-tiling of the bLW cores with the arm length of p-bp and the sticky end length of q-nt) assemblies, the arm can be overwound, resulting in a lefthanded twist, and can also be underwound, resulting in a right-handed twist. Chiral bLW-Ep/q tubes with either a right-handed curvature or a left-handed curvature can also be formed by the coupling of the intrinsic tile curvature and the arm twist. We were able to assign the chiral indices (n,m) to each tube using high-resolution AFM images, and therefore were able to estimate the tile curvature using a regular polygon model that approximated the transverse section of the tube. A deeper understanding of the integrated actions of different types of twisting forces on the DNA tubes will be extremely helpful in engineering more elaborate DNA nanostructures in the future.

For the past 40 years, the field of DNA nanotechnology has relied on the programmability of Watson-Crick base pair (bp) interactions to construct versatile DNA nanostructures and nanodevices ${ }^{1-8}$. The organization of $1 \mathrm{D}, 2 \mathrm{D}$, and 3D DNA nanostructures has been traditionally divided into two hierarchically coherent architectures: the weaving architecture and the tiling architecture. The weaving architecture relies on the winding of thread-like single-stranded DNAs into cross-linked double helixes by crossovers mainly three- 
and four-branched junctions, resulting in building blocks of stable tiles in nanoscale and organized lattices in macroscale, for example, double crossover (DX) tiles, point star junction tiles ${ }^{9-13}$, DNA origami ${ }^{3,14}$, and single-stranded tile and brick lattices ${ }^{15-17}$. Each tile can be viewed as having two main components: (1) a tile core surrounded by the scaffold strand with its boundary junctions, and (2) the outer overhangs carrying duplex stems with sticky ends for joining into the inter-tile arms. The tiling architecture refers to the assembly of DNA tiles into either finite or non-finite nanostructures by joining tiles with arms through sticky ended cohesion. In our current work, we term the joining with an arm length containing an even number of half-turns as E-tiling and that of an odd number of halfturns as O-tiling. The intrinsic curvature of a tile is a resultant of its weaving architecture, hereinafter specified as the intrinsic tile curvature, whereas the twist (or supercoiling) of an arm is generated from its tiling architecture, hereinafter specified as the arm twist. The coupling of both finely tunes the 2D DNA nanostructures into different morphologies and chiralities. In the periodic 2D DNA nanoconstructs, Bravais lattices can be abstracted, with tile cores as lattice points, joining arms as bonds, and the smallest repeating structures as unit cells. Thus, the 2D planar and tubular lattices in this work will be described via crystal terms and analyzed through concepts from crystal theory.

Since the double helix has a spring-like structure, the E-tiling architecture often generates DNA tubes and the O-tiling architecture produces planar DNA ribbons, a phenomenon attributed to the intrinsic curvatures of the DNA tiles ${ }^{18,19}$. E-tiling requires all the tiles to be aligned identically while O-tiling asks for the joined tiles to be aligned alternately. Assuming that the arms are straight and do not generate any torque along the axes of the arms, E-tiling will lead to the intrinsic curvature of each tile adding up to generate tubes, whereas O-tiling will produce planar ribbons due to the overall cancelation of the cumulative curvatures. Qualitative explanations regarding the tile curvatures have been proven to be successful in most cases. However, details regarding the structural and quantitative descriptions of the tile curvatures have been lacking such as the conformation of the tile (the addressable concave and convex faces), the curvature value with a geometrical definition, and the transition and transformation of the curvature with changing the length of the arms and/or the length of the sticky ends. The absence of such data in the design toolbox of DNA nanotechnology has constituted a hurdle to efficiently construct precise and diverse DNA nanostructures.

The design of curled DNA nanostructures with addressable surfaces follows a well-established strategy of periodic truncation (overwinding) or insertion (underwinding) in short duplex segments between adjacent Holliday junctions (HJs), which results in a net torsional strain on each segment, leading to the formation of either left-handed or right-handed constructs ${ }^{20-22}$. To describe the overwinding and underwinding strategy in more details, when the helical winding density in an arm is at 10.5-bp/turn (10.44-bp/turn to be precise $)^{22}$ of the canonical B-form of DNA, the arm is straight; when it is less than 10.5-bp/turn (overwinding), the arm gains a left-handed twist; and when it is larger than 10.5-bp/turn (underwinding), the arm gains a right-handed twist. Such twisting effects have been applied in DNA origami bundles and single stranded tile tubes ${ }^{23-27}$. However, DNA weaving and tiling architectures are integrated together in the two systems, where the cores and the arms cannot be clearly distinguished. A large number of E-tiling tubes have been assembled with the core-arm structure ${ }^{28-37}$, while very few reports mentioned about their addressable inside and outside surfaces, as well as the conformations of their component tiles ${ }^{33,34}$.

In the DAE-E tubes (E-tiling of DAE cores (DAE means an even number of half-turns of two antiparallel double helixes between double crossovers) $)^{33}$, six helix bundle ${ }^{38,39}$, and single-stranded tile nanoconstructs ${ }^{16,40}$, the universal polygon models have been applied to explain the formation of tubes. Such polygon models are based on the normal DNA winding criteria at the B-form including the match between the major and minor grooves at HJ crossovers by presupposing that all the helixes are rigid, tangent between adjacent helixes, and parallel to the tube axis. However, a precise physical description of the intrinsic tile curvature and its cooperation with the arm twist has not yet been illustrated clearly in the polygon models mentioned above.

Based on our previous works using small circular DNA molecules as scaffolds for construction of DNA tiles and nanostructures ${ }^{41,42}$, we folded a circular scaffold of 128 nucleotides (abbreviated as c128nt) into an H-shape and an X-shape, and obtained three weaving 
architectures to build (1) a traditional longitudinal weaving (LW) along the H-shaped scaffold to generate LW cores, LW-Ep/q and $\mathrm{LW}-\mathrm{O}_{\mathrm{p} / \mathrm{q}}$ nanostructures $\left(\mathrm{E}_{\mathrm{p} / \mathrm{q}} / \mathrm{O}_{\mathrm{p} / \mathrm{q}}\right.$ refers to the E-tiling/O-tiling architecture with the length of the arms at p-bp and the length of the sticky ends at q-nt), (2) a bridged longitudinal weaving (bLW) along the X-shaped scaffold by inserting a 10-bp helix at the center to yield bLW cores, bLW-Ep/q and bLW-O $/ \mathrm{q}$ nanostructures, and (3) a transverse weaving (TW) of the H-shaped scaffold to produce TW cores, TW-E $/ \mathrm{q}$ and TW-Op/q nanostructures.

In order to quantify the curvatures of the tiles, we first relied on the high-resolution AFM images to abstract the 2D Bravais lattices of centered rectangles for the designs and precisely measured their unit cell parameters. The E-tiling tubes were clearly the only adequate structures for the analysis of curvatures. Secondly, we described each specific tube with the chiral indices (n,m) $)^{43,44}$ by analyzing its high-resolution AFM images. Thirdly, we projected the unit tube possessing the chiral indices (n,m) onto its transverse section and approximated the projection to a regular polygon model (our analysis focused only on the two types of stable tubes, $\mathrm{n}=$ $2 \mathrm{~m}$ and $\mathrm{n}=\mathrm{m}$ ). The exterior angle of the regular polygon was assigned as the curvature of the tile (specified as the tile curvature). This curvature is also the global curvature of the tile (specified as the global tile curvature), which integrates the intrinsic tile curvature and the arm twist. The intrinsic tile curvature is defined as the curvature of a free tile in aqueous solution, which depends on its weaving architecture. Its measurement is nonetheless beyond our current capabilities. In practice, we could assign the tile curvature from an E-tiling tube - with an arm length of 21-bp and a sticky end length of 5-nt (specified as E21/5) - as the intrinsic tile curvature, which is based on the postulation that the $\mathrm{E}_{21 / 5} \mathrm{arm}$ is straight and does not generate any additional torque along the axis of the arm ${ }^{20}$. Under such a postulation, the intrinsic tile curvature represents exactly the intrinsic curvature of the tile core.

We relied on the biotin/streptavidin (SA) labeling technique and AFM imaging to define the configurations of the E-tiling tubes and thus the conformations of the DNA tiles, primarily for the minimally distorted tubes according to the B-form of DNA. We represent the chirality of a tube - along with the chirality of its component tiles - with either the right-hand or the left-hand grip rule. For demonstration purposes, with the thumb pointing towards the inside surface of a tube (i.e., the concave face of its component tile), the rotation direction of c128nt from 5' to 3' is followed by either the right-hand or the left-hand grip. For concise notation, we use

$l$ - or $r$ - as the prefix of the chiral indices $(\mathrm{n}, \mathrm{m})$ of a specific tube as $l-(\mathrm{n}, \mathrm{m})$ or $r-(\mathrm{n}, \mathrm{m})$ to represent its left-handed or right-handed chirality. Similarly, we identified the opposite faces of a tile by using $l$-face and $r$-face as labels. Moreover, the prefix of $l$ - or $r$ - in front of the $(n, m)$ indices indicates a clear 3D geometry of the tube, whereas $l$-face or $r$-face only identifies a specific $2 \mathrm{D}$ face of the tile.

\section{D lattices of longitudinally woven $(\mathrm{LW})$ tiles}

We applied the unified 2D Bravais lattices of centered rectangles to describe all the 2D DNA lattices originated from the c128ntderived tiles. For example, in the assembly model of Figure 1A(a), a compound unit cell of centered rectangle containing two tiles is defined by the mutually orthogonal basis vectors $\boldsymbol{a}$ and $\boldsymbol{b}$, with their respective unit lengths $a$ and $b$. For clear demonstration of the lattice structure and the tile conformation, a primitive cell of rhombus containing one tile, defined by the primitive vectors $c_{1}$ and $c_{2}$ with the unit length $c$ and the inter-angle $\varphi$, is superimposed. The theoretical estimations of $a, b, c$, and $\varphi$ in all the designs are described in Section S2 (data refer to Table S1). By achieving a lateral resolution of about $2.0 \mathrm{~nm}$ in the AFM images, we were able to precisely measure the unit cell parameters of each lattice, which confirmed our designs and were subsequently used for the numerical calculations (data refer to Tables 1 and S1).

Figure 1 illustrates the designs of both LW-E21/5 (panels A(a) and A(b)) and LW-O26/4 (panels B(a) and B(b)) and their assembly results (panels $A(c), A(d), B(c)$, and $B(d)$ ). In contrast with the general belief that the E-tiling architecture results in the formation of tubes, the LW-E21/5 lattices show mostly planar ribbons along with some nonhomogeneous wide tubes, and occasionally lengthy monolayer fibers (Figures $1 \mathrm{~A}(\mathrm{c}), 1 \mathrm{~A}(\mathrm{~d})$, and S12). The LW-O $26 / 4$ lattices exhibit mostly planar ribbons (Figures 1B(c), 1B(d) and S13). The assembly results indicated that the longitudinal weaving of H-shaped c128nt generated a tightly folded, crowded, rigid, 
and nearly planar LW core with minimal curvature, similar to our previously reported cDAO-c64nt system ${ }^{41}$. According to the traditional faceted crystal growth theory, lattice edges along the bond directions of $c_{1}$ and $c_{2}$ (the so-called flat faces) would appear thermodynamically, whereas edges along $\boldsymbol{a}$ and $\boldsymbol{b}$ (the so-called stepped faces) would occur dynamically. In the LW-E21/5 and LW-O26/4 designs, either $c_{1}$ or $c_{2}$ only has a small inter-angle (less than $20^{\circ}$ ) with respect to $\boldsymbol{a}$, and the aspect ratios of $a / b$ are larger than 1.5. Therefore, the growth of their 2D lattices was dynamically favored, and the lengthy edges of planar ribbons and the axes of tubes were mostly imaged along $\boldsymbol{a}$, belonging to the stepped faces.
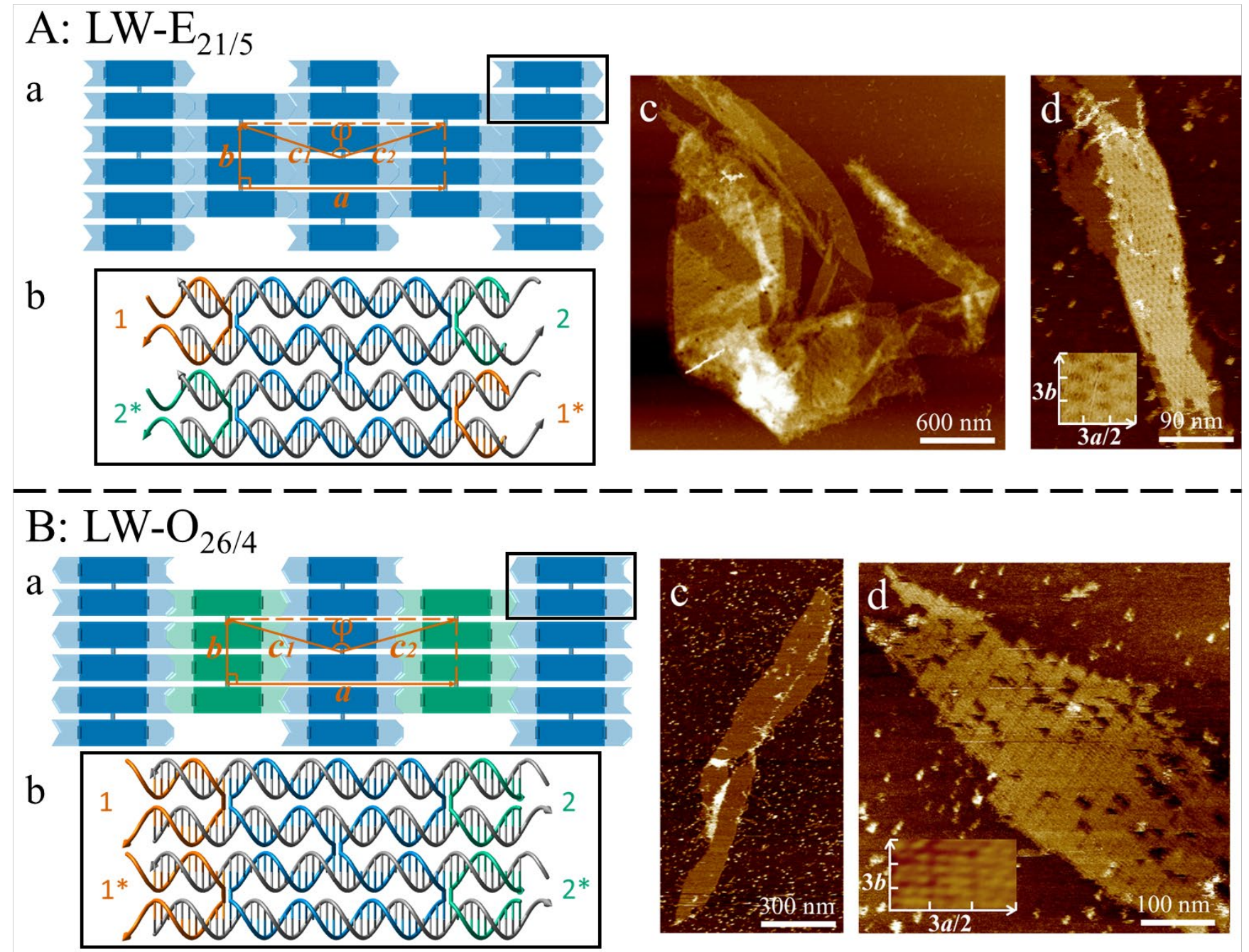

Figure 1. Longitudinal weaving and tiling architectures for the $\mathbf{L W}-\mathbf{E}_{21 / 5}$ and $\mathbf{L W}-\mathbf{O}_{26 / 4}$ systems. (A) LW-E21/5 assemblies: a) the Etiling assembly model with each brick colored in blue representing a tile with its $l$-face facing up, b) the helical tile model with the $l$-face facing up, corresponding to a brick framed in the rectangular box of (a); c and d) the respective zoomed-out and zoomed-in AFM images. (B) LW-O26/4 assemblies: a) the O-tiling assembly model with adjacent bricks along $\boldsymbol{a}$ colored in blue for the $l$-faces and in green for the $r$ faces; b) the helical tile model with the $l$-face facing up; c and d) the respective zoomed-out and zoomed-in AFM images. In all the helical tile models of this article, the blue strand c128nt acts as the central scaffold and rotates clockwise in the 5' $\rightarrow 3$ ' direction, thus, the tile's $l$ face always faces up, and its opposite $r$-face always faces down; the same numbered overhangs of $1,1 *$ or $2,2 *$ will cohere together to form an arm. In the zoomed-out AFM images of $\mathrm{A}(\mathrm{d})$ and $\mathrm{B}(\mathrm{d})$, each inset shows the organization of 9 tiles in a rectangle of length $3 a / 2$ and width $3 b$, clearly illustrating the individual cores and their cohesion regions of sticky ends. The measured lattice constants for the LW-E21/5 system 
are $a=34.6 \mathrm{~nm}, b=12.0 \mathrm{~nm}, c=18.3 \mathrm{~nm}, \varphi=142^{\circ}$; for the $\mathrm{LW}-\mathrm{O}_{26 / 4}$ system the values are $a=37.4 \mathrm{~nm}, b=12.0 \mathrm{~nm}, \mathrm{c}=21.1 \mathrm{~nm}, \varphi=$ $149^{\circ}$.

Since AFM imaging markers were not used on the LW-derived tiles, their densely woven 2D lattices, as well as the TW-E21/5 and TW-O26/4 assemblies (which are described later) were primarily imaged in fluid as brighter patches of planar ribbons, or blurred tubes without any defined texture (Figures $1 \mathrm{~A}(\mathrm{c}), 1 \mathrm{~B}(\mathrm{c}), 3 \mathrm{~A}(\mathrm{c})$, and 3B(b)). In order to obtain high-resolution images with defined appearances we employed two imaging techniques. 1) For the wide tubes, the top layer was sitting flat on the bottom layer and both layers electrostatically repulsed each other. The rhombus texture of the top layer was easily resolved with 1 to 3 scans, as shown in Figure $1 \mathrm{~A}(\mathrm{~d})$ and in the magnified insert. In comparison with the brick assembly model of Figure $1 \mathrm{~A}(\mathrm{a})$, each bright intersection point represents an LW core, each edge represents a joining arm of 21-bp, and each dim pit represents a rhombus center which was a little bit torn apart by repeated scans. 2) For the LW-O26/4 monolayer lattice which was strongly adsorbed on the hard substrate of mica, its high-resolution image in Figure 1B(d) was achieved after more than 4 scans. However, repeated scans removed some of the tiles and generated scattered empty pores in the lattice. Two hierarchical levels are shown in the structural textures. In the first level, the bright tile column and the dim fine stripe of the sticky ended cohesion (4-bp or $0.32 \mathrm{~nm} \times 4=1.3 \mathrm{~nm}$ wide) alternate with each other and are parallel to $\boldsymbol{b}$, having a periodic distance corresponding to the tile length of $a / 2$, shown in Figure $1 \mathrm{~B}(\mathrm{~d})$. In the second level, the magnification of the bright tile column in the insert of Figure 1B(d) shows the sub-details of the tiles, in which the a-parallel, bright, short bar, corresponding to either the upper half-part or the lower half-part of a tile (a segment of double double helixes), alternates with a dim slit, corresponding to the gap between the adjacent half-parts of the tiles. Moreover, the central HJ of the LW core was occasionally imaged as a bright dot. We hypothesize that the strong adsorption of the DNA helixes on the hard mica enabled the subdetails of the tiles to be resolved. The unit cell parameters for the LW-E21/5 and LW-O26/4 lattices listed in the caption of Figure 1 are in agreement with their theoretical estimates (Section S2 and Table S1). The high-resolution images also confirmed the correct assignment of the Bravais lattices of centered rectangles to both designs.

\section{Chiral indices and curvature evolution of the bLW-Ep/q tubes}

The strategies of central bridging to build 2D bLW-derived nanostructures are demonstrated in Figure 2. The insertion of a flexible 10-bp duplex forming two three-branch junctions provides more space and flexibility so that the bLW-derived tiles can regulate themselves more easily to fit within the lattices. The bLW-O26/4 assemblies (Figures 2A and S14) were composed overwhelmingly of tubes with scattered perimeters. The bLW core is X-shaped with two pairs of vertical angles of about $60^{\circ}$ and $120^{\circ}$, schematically drawn in both the brick model (Figure 2A(a)) and the helical model (Figure 2A(b)), which was deduced from the AFM images of Figures $2 \mathrm{~A}(\mathrm{c})$ and $2 \mathrm{~A}(\mathrm{~d})$. Figure $2 \mathrm{~A}(\mathrm{c})$ illustrates one of the widest tubes with a perimeter of $1.6-\mu \mathrm{m}$ (twice the width of the squashed double-layer), and Figure 2A(d) demonstrates the sub-details of the tile. In Figure 2A(d), two tiny faint pores at each intersection point can be seen separated by the 10-bp bridge, confirming our correct geometric assignment of the vertical angles of $60^{\circ}$ in the tile models of Figure 2.

In Figure 2B, we use three sets of chiral indices $(n, m)$ to specify the E-tiling DNA tubes. Such chiral indices have been previously used mostly in defining the structures of carbon nanotube ${ }^{43,44}$, and rarely been used in describing the structures of DNA tubes ${ }^{31}$. The Bravais unit cell is drawn at the bottom left corner of the hexagonal lattice background $\left(\varphi=120^{\circ}\right)$ which represents the bLW-Ep/q lattice with the $l$-faces of tiles facing up. Three sets of chiral indices $(n, m)$ are drawn to represent three specific unit tubes: $(8,4)$ in purple to represent a typical unit tube of bLW-E21/5, (3,3) in green to represent the unique unit tube of bLW-E31/7, and $(20,-20)$ in blue to represent a unit tube of the typical "giant" bLW-E32/6 tubes. The chiral indices $(\mathrm{n}, \mathrm{m})$ are abstracted from nc $1+$ m $c_{2}$, representing the chiral (or perimeter) vector $\boldsymbol{C h}(\mathrm{n}, \mathrm{m})$ originated from $\mathbf{O}$. For example, $(8,4)$ and $(3,3)$ are abstracted from $\boldsymbol{C}_{\boldsymbol{h}}(8,4)=8 \boldsymbol{c}_{\boldsymbol{1}}+4 \boldsymbol{c}_{2}$ and $\boldsymbol{C}_{\boldsymbol{h}}(3,3)=3 \boldsymbol{c}_{\boldsymbol{1}}+3 \boldsymbol{c}_{2}$, respectively. The unit tube of $(20,-20)$ is represented at the top right, in which vectors of $20 \boldsymbol{c}_{1}$ and $-20 \boldsymbol{c}_{2}$ are omitted for illustration purposes. The parameters of $c$ and $\varphi$ were measured directly from the AFM images and thus, the chiral indices (n,m) 
precisely defined many tube parameters, including the perimeter (diameter and radius), the helical angle (defined as the inter-angle either between $\boldsymbol{c}_{2}$ and $\boldsymbol{C h}(\mathrm{n}, \mathrm{m})$ or between $\boldsymbol{c}_{2}$ and $\left.\boldsymbol{T}\right)$, the axial periodicity $(\boldsymbol{T})$ (Section S3 and Figure S4), as well as the tile curvature. By combining both techniques of biotin/SA labeling and AFM imaging, we analyzed in detail the perimeters and configurations of the bLW- $\mathrm{E}_{21 / 5}$ tubes, and thus the curvatures and conformations of their component tiles in Figures $2 \mathrm{C}(\mathrm{a}-\mathrm{f})$. Figure $2 \mathrm{C}(\mathrm{a})$ shows the schematic brick model of the typical tube $(8,4)$ (top panel) and the model of its component tile (bottom panel) with a biotin label at overhang 2 (Two cohesion modes of sticky ends, shown in Figures 2C(a) and 2D(a), were examined for the bLW-E21/5 assemblies, as well as for other two assemblies of bLW-E20/4 and bLW-E22/6. We did not observe any difference between the results obtained from the two cohesion modes. Hereinafter the data from the two cohesion modes were combined together for analysis.). Figure $2 \mathrm{C}(\mathrm{b})$ provides a zoomed-out AFM image containing densely distributed tubes (top panel) and a zoomed-in image of a typical tube (bottom panel). Figure $2 \mathrm{C}(\mathrm{c})$ shows the distribution of 73 tubes assembled from more than 5 batches in 4 perimeter windows. The tubes appeared as mostly homogeneous, falling within the perimeter range between 110 and $270 \mathrm{~nm}$. A striking feature of the bLW-E21/5 tubes in all the zoomed-in images (Figures $2 \mathrm{C}(\mathrm{b}), 2 \mathrm{C}(\mathrm{d}), \mathrm{S} 4-9$, and $\mathrm{S} 15$ ) was that the two groups of $\boldsymbol{c}_{1-}$ and $\boldsymbol{c}_{2}$-frames were not symmetric. One group of frames were aligned nearly parallel to the tube axis whereas the other group were curled around the tube axis with a left-handed rolling. Figure $2 \mathrm{C}(\mathrm{d})$ shows the assembly model of the $l$-faced bricks with each tile carrying a biotin/SA label (top panel), and the zoomed-in AFM image of the inside surface of the tube (bottom panel). The ideal match of the background lattice overlain by the SA dots (each SA dot has a diameter around $5.5 \mathrm{~nm})^{37}$ between the real image and the model refers that the intrinsic tile curvature is left-handed.

We describe here our experimental approach. First, the tubes had to be in the open form to image both the background lattice and the overlying SA dots clearly, as shown in the bottom panel of Figure 2C(d) and in blocks II-IV of Figure S15. DNA tubes have been reported to be opened with repeated scans ${ }^{33}$. Herein, we report another experimental solution to prepare DNA tubes either in the open form or in both the open and closed forms by regulating the strength of the compressed air (Section S1.6 and Figure S3) when removing the sample drop. The top layers of the tubes were either partly or completely removed with water together due to the surface tension, whereas the bottom layers were strongly adsorbed and remained intact on the mica. The AFM scans were performed in either air or fluid mode. The open tubes exposing their inside surfaces outside are a requirement to define the direction of the curvature. For conciseness purposes, we only show the results of the biotin/SA labeling at the 5 '-end of the outer helper strand (green strand) of overhang 2 for the tubes of bLW-E21/5, bLW-E31/7, and bLW-E32/6 (additional AFM images are shown in Figures S15, S19, and S20). The other biotin/SA labeling results at overhang $1^{*}$ or overhang 1 are also shown in Figures S15-20. The in-situ binding of SAs on biotin-labeled lattices was carried out under a dilute SA solution according to previously reported protocols ${ }^{18,40}$. Although the biotinlabeled nucleotide was located at the buried $r$-face of the tiles in Figure 2C(d), a high yield of the biotin/SA binding (70 90 \%) was confirmed by AFM imaging, indicating that the empty space between the arms allowed biotins to bend away and stretch up from the mica surface, and bind SAs easily. From the massive biotin/SA dots overlying on the bLW-Ep/q monolayer lattices, we were able to precisely define in most cases the position of an SA dot relative to its host tile. For example, in the bottom panel of Figure 3C(d), the biotin/SA dots designed to sit on $c_{2}$-frames via cohesion of 2 and $2 *$ are aligned along the longitudinal frames. For each X-shaped tile with its center as a lattice point, the biotin/SA dot at overhang 2 via the acute angle to its two adjacent $\boldsymbol{c}_{1}$-frames is through clockwise rotation. Thus, the bLW-E21/5 tubes possess the left-handed curvature. Otherwise, if they have the right-handed curvature, the rotation direction from an SA-located $c_{2}$-arm via the acute angle to its two adjacent $c_{1}$-frames would be counterclockwise. Furthermore, we assigned that the longitudinal $c_{2}$-frames were parallel to and the $c_{1}$-frames curled with a left-handed rolling around the tube axis. In summary, we assayed over 60 individual bLW-E21/5 tubes at high-resolution from more than 5 batches with biotin/SA labels on overhangs $1,1^{*}$, and 2, all the imaging results supported the left-handed curvature (additional AFM images are in Figure S15). 
Quantitative analysis of the tile curvature from a specific tube relies on assigning the chiral indices ( $\mathrm{n}, \mathrm{m}$ ) to the tube. In practice, we are able to assign $(\mathrm{n}, \mathrm{m})$ to a perforated bLW-Ep/q tube based on its high-resolution AFM images illustrating the tube lattice in both the open and closed forms, such as in Figures S5-9. The zoomed-in AFM images indicated that around 90\% of the bLW-E21/5 tubes had 4, 5, and $6 c_{2}$-frames aligned parallel to the tube axis at the bottom layer, and a few other irregular tubes (around 10\%) either had $c_{2}$-frames clearly tilted against the tube axis or had wider perimeters. Combining the direct counting of the chiral indices (n,m) for the highly resolved tubes (Figures S4-9) with the numerical approximation of the perimeters of the tubes from the theory of chiral indices, we were able to assign the tubes with 4 bottom $c_{2}$-frames stuck on the mica to $l-(6,2), l-(6,3)$, and $l$-(7,3), the tubes with 5 bottom $c_{2}$-frames to $l$ - $(8,3), l-(8,4)$, and $l-(9,4)$, and the tubes with 6 bottom $c_{2}$-frames to $l-(10,4), l-(10,5)$, and $l-(11,5)(S e c t i o n s ~ S 3-$ $\mathrm{S} 5$, Figures S4-10, and Tables 1 and S2). The numerical calculation results illustrate that a cluster of $(\mathrm{n}, \mathrm{m}), \mathrm{where} \mathrm{n}=2 \mathrm{~m}, 2 \mathrm{~m}+1$, $2 \mathrm{~m}+2$, and $\mathrm{m}=3,4,5$, match the tube perimeters of Figure 2C(c) (Figure S10). The tile curvatures could be estimated from these clustered tubes. We took the most abundant bLW-E21/5 tube $l-(8,4)$ as an example to describe the quantitation approach. First, we considered that the geometry of a tile as the bending of its two halves to a dihedral angle along the edge passing through the tile center and being parallel to the tube axis, which could clearly be compared to a butt hinge with a pair of leaves bending along its shaft. It is reasonable to assume that the arms along $c_{1}$-frames in the bLW-E21/5 tube $l-(8,4)$ bend at their centers, where the sticky ended cohesion occurs, with the same curvature of the bLW core. Thus, we approximated the transverse section of the perforated tube $l-(8,4)$ to a regular 16-gon, as shown in Figure $2 \mathrm{C}(\mathrm{e})$. The physical appearance for the 16-gon was recognized as that the 16 vertices were projected from the unit tube of $(8,4)$ (Figure 2B) by 8 tile centers (lattice points) and 8 arm centers (lattice bond centers), and the 16 edges by 16 rigid half-lattice-bonds along $c_{1}$-frames. Therefore, the curvature $\theta$ of $l$ - $(8,4)$ was estimated as the exterior angle $360^{\circ} / 16$ $=22.5^{\circ}$, complemental to the dihedral angle schematically shown in Figure $2 \mathrm{C}(\mathrm{f})$. Similarly, a 12-gon was approximated from the narrowest tube $l-(6,3)$ and a 24 -gon from the widest tube $l-(12,6)$, and their tile curvatures were estimated at $30^{\circ}$ and $15^{\circ}$, separately. From the above approximation approaches, the intrinsic tile curvature of the bLW-E $21 / 5$ tubes was estimated to be $22.5^{\circ} \pm 8^{\circ}$.

To investigate how the overwinding and underwinding of the arms influence the tile curvature, we tested the overwound bLW-E20/4 assembly (the winding density of the arm at $10 \mathrm{bp}$ (20/2) is lower than $10.5 \mathrm{bp}$ ) and the underwound bLW-E22/6 assembly (the winding density of the arm at $11 \mathrm{bp}(22 / 2)$ is higher than $10.5 \mathrm{bp})$. Both types of tubes had lower yields than the B-form DNA tubes of bLWE21/5 because either the decrease or the increase of one base-pair in the arm from 21-bp, the arm length of the canonical B-form of DNA, could disturb the formation of the tubes to some degree. The biotin/SA labeling results showed that both tubes had the lefthanded curvatures (more than 10 individual tubes from 3 batches were measured for each design, as shown in Figures S16 and S17). The formation of the left-handed bLW-E20/4 tubes was driven by the left-handed twisting force emanated from both the intrinsic tile curvature and the overwinding of the arm. While in the bLW-E22/6 tubes, although a right-handed twist of the arm could be generated from both the underwinding of the arm and the sticky ended cohesion of 6-bp, the left-handed twist originated from the intrinsic tile curvature was still stronger, thereby resulting in the left-handed tubes. The bLW-E20/4 tubes were composed of $l$-(4,2) in majority and $l-(6,3)$ in minority (Figures S16), and the bLW-E22/6 tubes distributed from $l-(5,5)$ to $l-(9,9)$, among which $l-(7,7)$ was the most abundant (Table S3 and Figure S17). Additionally, not like the bLW-E21/5 and bLW-E20/4 tubes with two types of asymmetric frames, both $c_{1}$ - and $c_{2}$-frames in the bLW-E22/6 tubes were symmetric and they should play an equal framing role.

We also extended the arm length to three turns and tested the three designs of bLW-E31/5, bLW-E31/7, and bLW-E32/6. Similar to the bLW-E20/4 tubes, the overwound bLW-E31/5 design (the winding density of the arm at $10.3 \mathrm{bp}(31 / 3)$ is lower than10.5 bp) generated the typical tubes of $l-(8,4), l-(10,5)$, and $l-(12,6)$ with a moderate yield, of which the left-handed curvature was defined from the biotin/SA labeling results ( 8 individual tubes from 2 batches were measured, as shown in Table S4 and Figure S18).

The bLW-E31/7 assemblies lead to uniform tubes of $r$ - $(3,3)$ in high yield and with high quality, as shown in Figures $2 \mathrm{D}(\mathrm{a}-\mathrm{d})$ and in block I of Figure S19. Investigation of the tile conformation with the biotin/SA labeling technique was challenging because the 
perimeter of the unique bLW-E31/7 tube $r$ - $(3,3)$ reached the lower width limit of an open monolayer strip for defining the position of an SA dot relative to its host tile (Section S1.7). With much effort, we imaged more than 100 tubes from 5 batches of samples, but we were only able to clearly define the right-handed curvature for 15 tubes, as shown in Figure 2D(c) and in blocks II and III of Figure S19. With the similar approximation approach to $l-(8,4)$, projection of the unit tube of $r-(3,3)$ to its transverse section generated a regular 12-gon. Thus, the global tile curvature of the bLW-E31/7 tube $r$ - $(3,3)$ was estimated as $30^{\circ}$ with the dihedral edge along $\boldsymbol{a}$, which is schematically shown in Figure $2 \mathrm{D}(\mathrm{d})$.

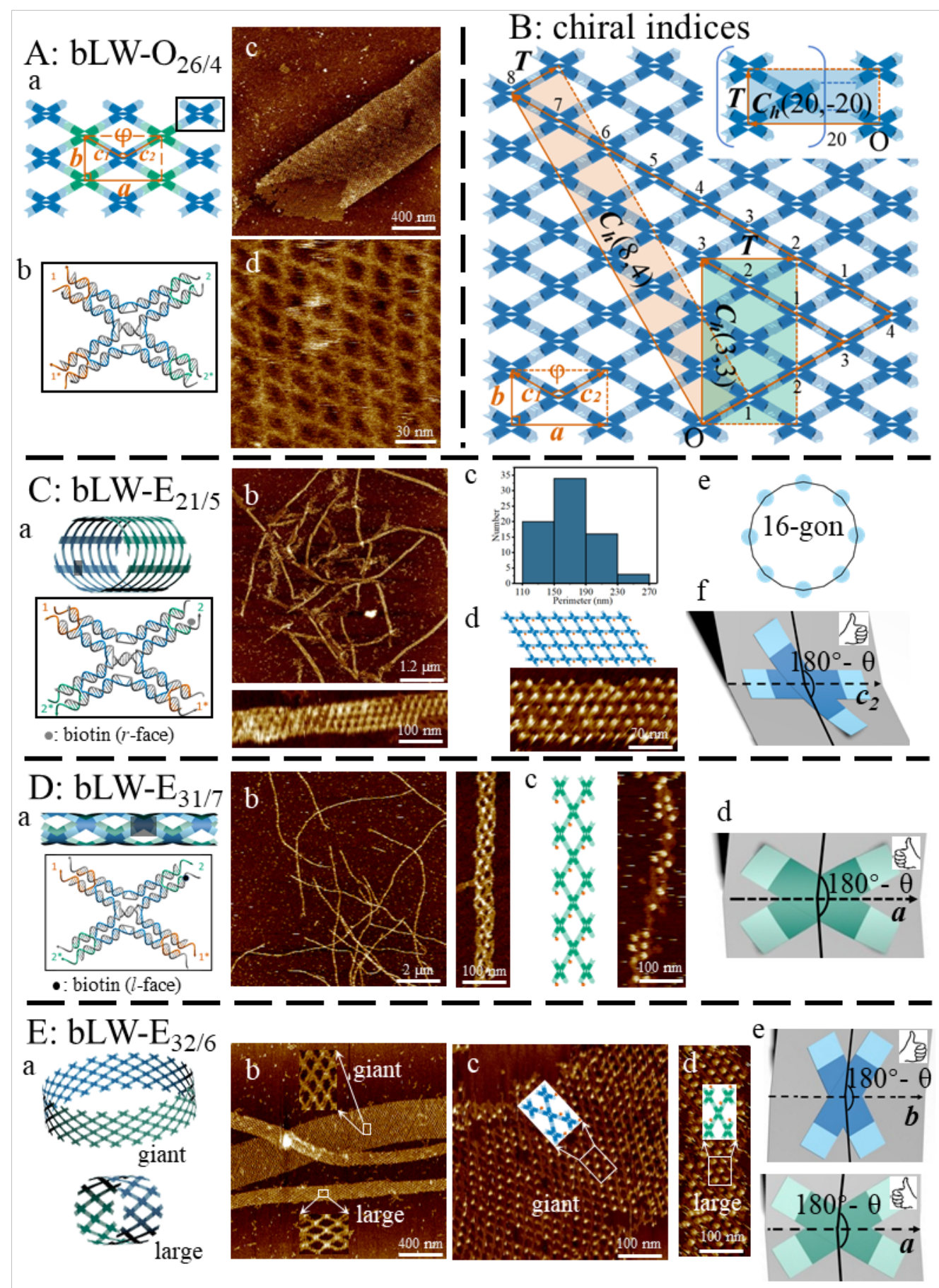

Figure 2. Bridged longitudinal weaving and tiling architectures and curvature transformation in bLW-Ep/q tubes. (A) bLW-O26/4 assemblies: a) the O-tiling assembly model, b) the helical tile model, c and d) the respective zoomed-out and zoomed-in AFM images. (B) 
Three sets of the chiral indices $(n, m)$ describing the tiling structures of 3 typical E-tiling tubes. An open unit tube of $(8,4)$ shadowed in gold with its tube axis along $c_{2}$ describes one of the typical bLW-E21/5 tubes, an open unit tube of $(3,3)$ shadowed in green with its tube axis along $\boldsymbol{a}$ describes the unique bLW- E31/7 tube, and an open unit tube of $(20,-20)$ shadowed in blue with its tube axis along $\boldsymbol{b}$ describes one of the typical "giant" bLW-E32/6 tubes. (C) bLW-E21/5 tubes: a) the tube model of $l$ - $(8,4)$ with the $l$-faces of tiles inside and the $r$-faces of tiles outside (top panel) and the tile model with a biotin label at overhang 2 pointing toward its $r$-face (bottom panel), b) the zoomed-out (top panel) and zoomed-in (bottom panel) AFM images of the bLW-E21/5 tubes, c) 73 tubes distributed in 4 perimeter windows, d) the assembly model with each tile carrying a biotin/SA label (a red star represents an SA) at overhang 2 (top panel) and its corresponding zoomed-in AFM image of the tube's inside surface (bottom panel), e) approximation of the transverse section of the tube $l$ - $(8,4)$ with a regular 16 -gon, f) the intrinsic tile curvature represented with the dihedral $\angle \boldsymbol{c} 2$ following the left-hand grip rule. (D) bLW-E31/7 tubes: a) the tube model of $r$-(3,3) with the $l$-faces of tiles outside and the $r$-faces of tiles inside (top panel) and the tile model with a biotin label at overhang 2 pointing toward its $l$-face (bottom panel), b) the zoomed-out (left panel) and zoomed-in (right panel) AFM images, c) the assembly model with each brick carrying a biotin/SA label at overhang 2 (left panel) and its corresponding zoomed-in AFM image of the inside surface of the tube $r$-(3,3) (right panel), d) the global tile curvature of $r$ - $(3,3)$ represented with the dihedral $\angle \boldsymbol{a}$ following the right-hand grip rule. (E) bLW-E32/6 tubes: a) the "giant" tube model of $l-(20,-20)$ (top panel) and the "large" tube model of $r$ - $(10,10)$ (bottom panel) with their tile model of bLW-E32/6 possessing a biotin label at overhang 2 similar to the tile model of bLW-E31/7 in Figure 2D(a) omitted, b) a zoomed-out AFM image showing both "large" and "giant" tubes and their corresponding zoomed-in images in insets illustrating different tube axes, c) a zoomed-in image of the inside surface of a "giant" tube overlain by biotin/SA dots, corresponding to the assembly model with each $l$-faced tile carrying a biotin/SA dot at overhang 2 (inset), d) a zoomed-in image of the inside surface of a "large" tube overlain by biotin/SA dots, corresponding to the assembly model with each $r$-faced tile carrying a biotin/SA dot at overhang 2 (inset), e) the global tile curvature of the "giant" tube represented with the dihedral $\angle \boldsymbol{b}$ following the left-hand grip rule (top panel) and that of the "large" tube with the dihedral $\angle \boldsymbol{a}$ following the right-hand grip rule (bottom panel).

The bLW-E32/6 tubes are shown in Figures 2E(a-e) with schematic drawings, AFM images, and the analysis results of curvatures. Much different from the previous designs which generated the same type of tube structures for each design, two different types of "giant" (a schematic of $l-(20,-20)$ at the top panel of Figure 2E(a)) and "large" (a schematic of $r-(10,10)$ at the bottom panel of Figure 2E(a)) tubes were imaged in high yield and with high quality (Figure 2E(b) and block I of Figure S20, Tables S5 and S6). The "giant" tubes grew along $\boldsymbol{b}$, whereas the "large" tubes grew along $\boldsymbol{a}$. Because both tubes' perimeters were large enough, they were easy to be opened to well-organized 2D monolayer lattices. Their tile conformations were easily defined with the biotin/SA labeling technique (over 20 individual tubes of each type from more than 3 batches were imaged). The "giant" tubes had the left-handed curvature (Figure 3E(c) and blocks II and IV of Figure S20), whereas the "large" tubes possessed the right-handed curvature (Figure 3E(d) and blocks III and V of Figure S20). The global tile curvature of the most abundant "giant" tube $l-(20,-20)$ was estimated at $4^{\circ}$ from a regular 80-gon (top panel of Figure $2 \mathrm{E}(\mathrm{e})$ ), and the global tile curvature of the most abundant "large" tube $r$-(10,10) at $9^{\circ}$ from a regular 40-gon (bottom panel of Figure 2E(e)).

In Table 1, we summarized the linear and angular lattice parameters $(a, b, c$, and $\varphi)$ and the parameters of the most abundant tube for each bLW-Ep/q assembly (the chiral indices $(n, m)$, the perimeter $(C)$, the tile curvature $(\theta)$, and the length range of tubes).

Table 1. Lattice parameters and tube parameters of the most abundant tube for each bLW-E/q design. ${ }^{\#}$

\begin{tabular}{|c|c|c|c|c|c|c|c|c|}
\hline Tube & $a / \mathrm{nm}$ & $b / \mathrm{nm}$ & $c / \mathrm{nm}$ & $\varphi /{ }^{\circ}$ & $\boldsymbol{C h}(\mathrm{n}, \mathrm{m})$ & $\mathrm{C} / \mathrm{nm}$ & $\theta($ dihedral edge $)$ & length/ $\mu \mathrm{m}$ \\
\hline bLW-E $E_{20 / 4}$ & 34.4 & 21.9 & 20.4 & $115^{\circ}$ & $l-(4,2)$ & 74.3 & $45^{\circ}\left(c_{2}\right)$ & $2.0-5.0$ \\
\hline bLW-E $E_{21 / 5}$ & 35.1 & 23.7 & 21.2 & $112^{\circ}$ & $l-(8,4)$ & 158.7 & $22.5^{\circ}\left(c_{2}\right)$ & $3.0-7.0$ \\
\hline $\mathrm{bLW}-\mathrm{E}_{22 / 6}$ & 36.3 & 22.7 & 21.4 & $116^{\circ}$ & $l-(7,7)$ & 158.9 & $12.9^{\circ}(a)$ & $2.0-5.0$ \\
\hline
\end{tabular}




$\begin{array}{lcccccccc}\mathrm{bLW}-\mathrm{E}_{31 / 5} & 41.2 & 25.7 & 24.3 & 116^{\circ} & l-(10,5) & 219.0 & 18^{\circ}\left(\boldsymbol{c}_{2}\right) & 4.0-7.0 \\ \begin{array}{l}\mathrm{bLW}-\mathrm{E}_{31 / 7} \\ \mathrm{bLW}-\mathrm{E}_{32 / 6}\end{array} & 41.9 & 24.2 & 24.2 & 120^{\circ} & r-(3,3) & 72.7 & 30^{\circ}(\boldsymbol{a}) & 6.0-10.0 \\ \begin{array}{l}\text { giant) } \\ \begin{array}{l}\mathrm{bLW}-\mathrm{E}_{32 / 6} \\ \text { (large) }\end{array}\end{array} & 42.3 & 24.9 & 24.5 & 119^{\circ} & l-(20,-20) & 844.4 & 4.5^{\circ}(\boldsymbol{b}) & 4.0-8.0 \\ \end{array}$

\# For all the 6 designs, we choose the most abundant tube of each design for demonstration. The tube perimeter $\mathrm{C}$ was calculated as $\mathrm{C}=c \sqrt{n^{2}+m^{2}+2 n m \cos \varphi}$ (Section S3). The tile curvature $\theta$ correlated with the dihedral edge of the tile indicated in the bracket was estimated according to the following approximation rule: For each bLW-Ep/q tube represented with $(\mathrm{n}, \mathrm{m})$, when $\mathrm{n}=2 \mathrm{~m}, \theta$ was calculated as the exterior angle of a regular 2n-gon; when $\mathrm{n}=|\mathrm{m}|, \theta$ was calculated as the exterior angle of a regular $4 \mathrm{n}$-gon.

As shown in the bLW-Ep/q tubes, the global tile curvature was influenced by many factors such as the intrinsic tile curvature, the length of the arm, the length of the sticky end, and the orientation of the tile $v s$ the tube axis. We simplified the multiple factors into a model in which the tube configuration was tuned by two twisting forces, one originated from the intrinsic tile curvature and the other emanated from the arm twist which was regulated by both the length of the arm and the length of the sticky end. The arm twist was roughly predicted by combining the overwinding/underwinding of the arm and the sticky ended cohesion although its precise control still needs further investigation ${ }^{47,48}$. Our experimental results provided the following reasonable deductions for the arm twist: 1) The extra twisting force generated from the weak sticky ended cohesion of 4 or 5-bp could be ignored. In this case, the arm twist was governed by the overwinding, normal winding, or underwinding of DNA double helix. 2) By strengthening the sticky ended cohesion to either 6 or 7-bp, an extra right-handed force was generated, thus, the arm twist was tuned by both the length of the arm and the length of the sticky end. The tube configuration (stemming from the global tile curvature) being either right-handed or lefthanded was determined by the coupling of the intrinsic tile curvature and the arm twist. We hypothesize the following mechanisms for the flip-over of the left-handed chirality governed by the intrinsic tile curvature of the bLW cores to the right-handed chirality directed by the arm twist. First, the formation of the unique bLW-E31/7 tube $r-(3,3)$ was governed by the right-handed twist of the arms generated from the sticky ended cohesion of 7-bp rather than by the left-handed twisting force yielded from the intrinsic tile curvature and the weak overwinding of the arm (the winding density 10.3 (31/3) bp/turn is less than $10.5 \mathrm{bp} /$ turn). Secondly, the simultaneous growth of both "giant" and "large" tubes from the bLW-E32/6 assemblies could be interpreted as follows: 1) Both the overwinding of the arm (the winding density 10.7 (32/3) bp/turn is bigger than $10.5 \mathrm{bp} /$ turn) and the sticky ended cohesion of 6-bp generated an integral right-handed twisting force. 2) The intrinsic curvature of the bLW core produced a left-handed twisting force. 3) A delicate balance should exist between the two opposite twisting forces at the initial assembly stage, which could drive the tile oligomers to form saddle-like structures (Section S6 and Figure S11). In the saddle-like oligomers, the deeper curvature surrounding $\boldsymbol{a}$ was driven by the right-handed twisting force and the other shallower curvature surrounding $\boldsymbol{b}$ by the left-handed twisting force. 4) With further growth, depending on which curled surface closed first to form a ring, as a tube seed, the ring ended up growing epitaxially to a full tube. The saddle-like structural model for the tile oligomers was strongly supported by two evidences. 1) The growth direction or the tube axis of the "large" tubes along $\boldsymbol{a}$ is perpendicular to that of the "giant" tubes along $\boldsymbol{b}$. 2) The global curvatures of the "large" tubes are right-handed, opposite to the left-handed curvatures of the "giant" tubes. We named the specifically paired group of "large" and "giant" tubes as "orthogonal" tubes. The phenomenon of tube-widening with $\boldsymbol{b}$ as the tube axis had also been observed in the DAE-E tubes. However, the authors addressed that the formation mechanism and the tube configuration were not clearly described ${ }^{33}$. In our case, the saddle-like model reasonably explains how to overcome the higher energy barrier and thus to form the "giant" ring as a seed which finally grows up epitaxially to a "giant" tube. The "giant" tubing phenomenon and the flip-over effect appeared in 
the bLW- $\mathrm{E}_{\mathrm{p} / \mathrm{q}}$ designs with the arm length at three full turns, but not at two full turns, indicating that the arm flexibility might play a role.

\section{D Lattices of transversely woven (TW) tiles}

Figures $3 \mathrm{~A}$ and $3 \mathrm{~B}$ show the transverse weaving of the H-shaped c128nt scaffold with 6 helper strands to a stable TW core, and further the O-tiling architecture to planar TW-O26/4 lattices and the E-tiling architecture to TW-E21/5 tubes. In the TW core, each helper strand weaves in the transverse direction over and under every other scaffold strand 4 times, forming the densest HJs in 2D DNA lattices with a weaving density of $2 \mathrm{HJ}$ s per turn ${ }^{45}$. Compared with the LW core having totally 5 HJs and the bLW core having totally $4 \mathrm{HJ}$, the TW core possesses totally $12 \mathrm{HJs}$.

The O-tiling architecture of TW-O26/4 provided planar handkerchief-like lattices with a maximum size up to $4 \times 4 \mu m^{2}$, shown in Figures $3 \mathrm{~A}(\mathrm{c})$ and S21. To achieve high-resolution images with individual tile textures, as shown in Figures 3A(d) and S21, more than 4 scans were needed. However, repeated scans moved some tiles away and left dim pores clearly in the lattice. In contrast with the LW-E21/5 and LW-O26/4 ribbon-like lattices having ragged edges (stepped faces) along the longitudinal direction $\boldsymbol{a}$, the TW-O26/4 lattices presented sharp vertices and straight edges (flat faces) along both $c_{1}$ and $c_{2}$ directions due to the strong bond with three double helixes integrated together as an arm via sticky ended cohesion ${ }^{46}$. As shown in Figure $3 \mathrm{~A}(\mathrm{~d})$, with both $\boldsymbol{c}_{1}$ and $\boldsymbol{c}_{2}$ assigned to be along the sharp edges, $\boldsymbol{a}$ and $\boldsymbol{b}$ are easily defined.

The E-tiling architecture of TW-E21/5 shows relatively homogenous tubes (Figure 3B(b) and block I of Figure S22). Although the TW core has the highest HJ density, formation of tubes instead of planar lattices indicates that the TW core has an intrinsic curvature, probably due to the lack of a central HJ and therefore the bending of both half-parts along $\boldsymbol{b}$ passing through the TW core center. Repeated scans of a tube more than 4 times opened the tube and revealed individual tile textures (Figure 3B(c) and block I of Figure S22). As shown in Figure 3B(c), parts of the top layers are torn open along the flat face $c_{1}$ or $c_{2}$. The tube axis is along $\boldsymbol{a}$ (i.e. the arm helix extension direction) because it is thermodynamically favored. Counting the lattice points (or tiles) along both $c_{1}$ and $c_{2}$ on the top layer of the tube in Figure 3B(c), we could assign the chiral indices $(5,5)$ to it.

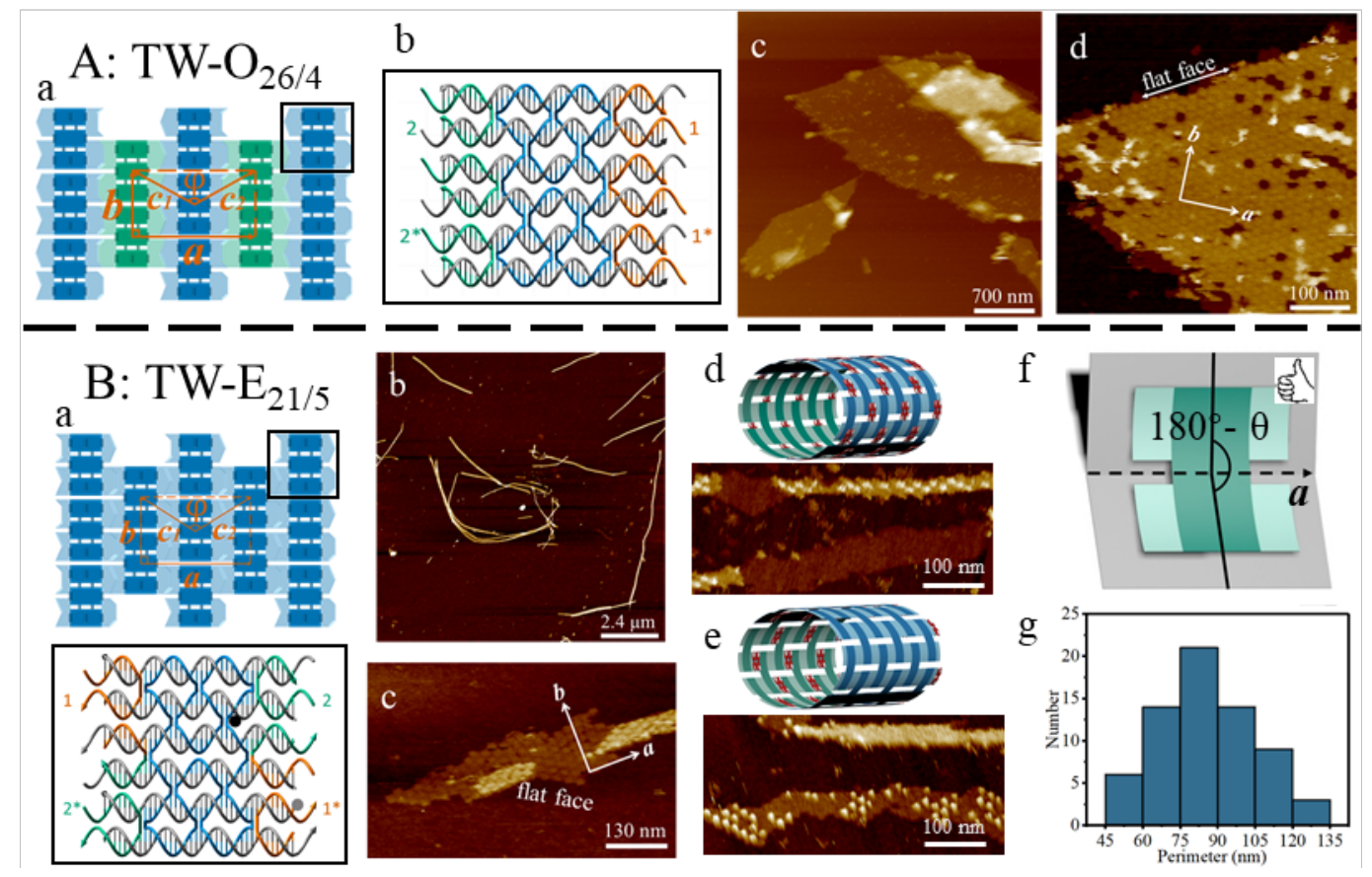

Figure 3. Transverse weaving and tiling architectures of the TW-O26/4 and TW-E21/5 systems. (A) TW-O26/4 assemblies: a) the O-tiling assembly model, b) the helical tile model, in which the two central helixes are 20-bp and the four upper and lower helixes are 22-bp in length 
within the core along $\boldsymbol{a}, \mathrm{c}$ and d) the zoomed-out and zoomed-in AFM images with lattice constants of $a=30.6 \mathrm{~nm}, b=14.7 \mathrm{~nm}, c=17.0$ $\mathrm{nm}$, and $\varphi=129^{\circ}$. (B) TW-E21/5 assemblies: a) the assembly model (top panel) and the helical tile model with a 5'-biotin label (gray dot) pointing toward its $r$-face and an iBiodT (dark dot) pointing toward its $l$-face (bottom panel), b and c) the zoomed-out and zoomed-in images with lattice constants of $a=27.3 \mathrm{~nm}, b=14.3 \mathrm{~nm}, c=15.3 \mathrm{~nm}$, and $\varphi=128^{\circ}$, d) the assembly model of a tube $(r$ - $(6,6)$ as an example here) with each tile's $l$-face at the outside surface of the tube possessing a biotin/SA label (top panel) and a corresponding zoomed-in image illustrating the SA dots binding only on the surface of the double-layers but not the monolayers (bottom panel), e) the assembly model of a tube $(r-(6,6)$ as an example here) with each tile's $r$-face at the inside surface of the tube possessing a biotin/SA label (top panel) and a corresponding zoomed-in image illustrating the SA dots binding only on the surface of the monolayers but not the double-layers (bottom panel), f) the intrinsic tile curvature of the TW-E21/5 tubes represented with the dihedral $\angle \boldsymbol{a}$ following the right-hand grip rule, (g) 67 tubes distributed in 6 perimeter windows.

The tile conformation is defined with the in-situ biotin/SA labeling technique too. Different from the perforated bLW-Ep/q tubes, the TW-E21/5 assemblies were sealed tubes without leaving any space between the tiles. In this case, the biotin orientation on micasupported lattices was key for the in-situ binding of SAs. When biotins were displayed on the top of a TW-E21/5 lattice, SAs bound to biotins efficiently, whereas when biotins were buried under a TW-E21/5 lattice, no binding could occur due to the steric hindrance. We applied three biotin-labeling strategies for the TW-E21/5 tile: 1) an iBiodT label at overhang 2 pointing toward the $l$-face, 2) a biotin label at the 5 '-end of a helper strand at overhang $1 *$ pointing toward the $r$-face, 3 ) both types of biotin labels at the $r$ - and $l$-faces, as shown in the tile model of Figure 3B(a) (bottom panel). After in-situ binding, the biotin labels displayed on the l-faces bound SAs only at the top of the sealed tubes (seen as double-layers) but not on the open tubes (seen as monolayers), as shown in Figure 3B(d) and block II of Figure S22; the biotin labels displayed on the $r$-faces bound SAs only on the monolayers but not on the double-layers, as shown in Figure 3B(e) and block III of Figure S22; the biotin labels displayed on both the $r$ - and $l$-faces bound SAs on both monolayers and double-layers, as shown in block IV of Figure S22. For each labeling design, we imaged more than 30 individual tubes from 3 separate batches. All the imaging results supported the right-handed curvature of the TW-E21/5 tiles. The same biotin/SA binding strategy for addressing the conformation of the TW core was also applied to the well-known DAE-E21/5 tubes as a control experiment. The right-handed curvature was deduced for the DAE-E21/5 tubes with the biotin/SA labeling technique (Figure S23), in good agreement with the results obtained from other techniques ${ }^{33,34}$. The successful definition of the tile conformation in the E-tiling tubes of this work confirmed the ease and usefulness of the in-situ biotin/SA labeling technique to define the stereostructures of DNA tubes and tiles.

We measured the perimeters of 67 tubes in more than 5 different batches by doubling the width of the double-layer. The abundances of the tubes at different perimeter windows are plotted in Figure $3 \mathrm{~B}(\mathrm{~g})$, indicating a nearly normal distribution from $45 \mathrm{to} 135 \mathrm{~nm}$. By combining the direct counting of the chiral indices $(n, m)$ obtained from the high-resolution AFM images and the numerical approximation method shown in Sections S3-S5, we assigned the most abundant tubes $(21 / 67=31.3 \%)$ at the perimeter window of $75.0 \sim 90.0 \mathrm{~nm}$ to $r-(6,6)$, the second most abundant tubes $(14 / 67=21 \%)$ at $60.0 \sim 75.0 \mathrm{~nm}$ to $r-(5,5)$ and at $90.0 \sim 105.0 \mathrm{~nm}$ to $r-(7,7)$, and the less abundant tubes at $45.0 \sim 60.0 \mathrm{~nm}$ to $r-(4,4)(6 / 67=9.0 \%)$, at $105.0 \sim 120.0 \mathrm{~nm}$ to $r-(8,8)(9 / 67=13.4 \%)$, and at $120.0 \sim 135.0$ $\mathrm{nm}$ to $r$ - $(9,9)(3 / 67=4.5 \%)$ (Table S7). Since the helixes of the TW-E21/5 tubes are closely juxtaposed without leaving any space between them, and each arm is composed of treble double helixes which are parallel to the tube axis, we hypothesize that the arm is straight and no bending occurs at the sticky ended cohesion regions. Therefore, the transverse section of the most abundant tube $r$ $(6,6)$ was approximated to a regular 12-gon, that of the narrowest tube $r$ - $(4,4)$ to an 8 -gon, and that of the widest tube $r$ - $(9,9)$ to an 18-gon. Therefore, the intrinsic tile curvature of the TW-E21/5 tubes was estimated to be at $30^{\circ} \pm 15^{\circ}$, which is schematically represented in Figure 3B(f).

\section{Discussion}


We grew tubes using a very slow annealing process of 70 hours for a better thermodynamic control of the assembly process. The chiral indices ( $\mathrm{n}, \mathrm{m})$ assigned to the tubes in this work belong to two main clusters of 1$) \mathrm{n} \approx|\mathrm{m}|$ and 2 ) $\mathrm{n} \approx 2 \mathrm{~m}$ where $0 \leq|\mathrm{m}| \leq \mathrm{n}$. The two types of chiral indices should represent the stablest tube structures at the global energy minimum. The formation of the symmetric tubes represented with $(\mathrm{n}, \mathrm{m})$ where $\mathrm{n} \approx|\mathrm{m}|$ indicated that both $\boldsymbol{c}_{1}$ and $\boldsymbol{c}_{2}$-frames played the same framing role. While the formation of the specific asymmetric tubes represented with $(\mathrm{n}, \mathrm{m})$ where $\mathrm{n} \approx 2 \mathrm{~m}$ bLW-E21/5, bLW-E20/4, and bLW-E31/5 assemblies demonstrated that the intrinsic mechanics of the tiles played a role. Other tubes of $(\mathrm{n}, \mathrm{m})(0 \leq \mathrm{m} \leq \mathrm{n})$ deviating from $\mathrm{n} \approx 2 \mathrm{~m}$ and $\mathrm{n} \approx \mathrm{m}$ would possess higher deformation energies. Thereby they were not thermodynamically favored but dynamically controlled, such as trapping at the initial assembly stage as an intermediate ring which ended up growing epitaxially to a full tube. In fact, we imaged a very few of these tubes for most of the designer tubes examined. Lattice dislocations also occurred in most of the tubes, causing gradual changes of perimeters along the tube longitude. This deformation effect was found in the "giant" bLW-E32/6 tubes with a higher probability than in other narrower tubes.

The semi-quantification of the tile curvatures depends on the postulation of how the arm bends. We take the well-known DAE-E21/5 tubes as an example here for discussion. The perimeters of the normal DAE-E21/5 tubes tiled up symmetrically vary from 4 to 10 tiles ${ }^{33}$, which are assigned to the chiral indices of $r-(4,4)$ to $r$ - $(10,10)$ correspondently. If we adapt the well-accepted postulation that the arm is straight and parallel to the tube axis, the transverse projection of $r-(4,4)$ is approximated to an 8 -gon, and that of $r$ - $(10,10)$ to a 20 gon, thereby the intrinsic curvature of the DAE core is estimated between $18^{\circ}$ and $45^{\circ}$. If we postulate that the arms bend with the same curvature of the DAE tile cores, the transverse projection of $r-(4,4)$ is approximated to a 16 -gon, and that of $r$ - $(10,10)$ to a 40 gon, thereby the curvature of the DAE core is estimated between $9^{\circ}$ and $22.5^{\circ}$. The variable curvatures for the same type of tiles in a limited range is understandable because DNA is considered as the spring-like structure.

The O-tiling architecture often generates both planar ribbons and nonhomogeneous tubes, like in the well-known DAE-O26/4 System² . In other special cases such as in our bLW-O26/4 system, the O-tiling architecture produces mostly nonhomogeneous tubes. This result has been explained as the decrease of the global free energy of the system. From the viewpoint of DNA mechanics, we suggest that the phenomenon of flip-over of the curvature might somehow account for the tendency of the O-tiling assemblies to generate tubes. Since the intrinsic tile curvature always remains, we could compare the flip-over of the conformation of the bLW core to the flipover of the curvature of a contact lens. Once a ring seed randomly forms in the bLW-O26/4 assemblies, the epitaxial extension of the tube lattice from the ring seed could force the oppositely curved tiles to switch to the same curved ones. Rationally, with an appropriate rigidity, the coupling of a shallow intrinsic tile curvature with a weak arm twist could generate either large planar lattices or wide tubes with high probability. On the contrary, the coupling of a deep intrinsic tile curvature with a strong arm twist could probably yield ill-behaved fragments of tile oligomers, narrower tubes and fibers, rather than the well-behaved DNA lattices. Other couplings between the two extremes could generate moderate structures in a complicated way.

In summary, we not only realized three weaving architectures to construct three new tile cores, LW, bLW, TW, and their 2D lattices, but we also deciphered each tube's configuration and its component tile's conformation with the biotin/SA labeling technique. By abstracting the 2D Bravais lattice of centered rectangle for each assembly, we introduced the chiral indices for each tube to quantify its tile curvature. We classified the twisting forces producing tubes via the E-tiling architecture into two types: one generated from the intrinsic tile curvature and the other emanated from the arms. The coupling of these two forces allowed us to understand the formation mechanisms of the DNA tubes into a plethora of different shapes, diameters, and configurations. Such detailed investigations of the DNA tile curvatures including structure and quantitation will on one hand be helpful in the future for engineering DNA nanostructures in high yield and with high quality, and for realizing specific physicochemical properties and biological functionalities with the DNA chiral assemblies on the other hand.

\section{ASSOCIATED CONTENT}

Supporting Information available. experimental methods, a discussion about the theoretical estimation of lattice linear and angular constants, a discussion about the tube parameters calculated from the chiral indices $(n, m)$, a discussion about the assignment of the chiral indices 
$(n, m)$ to a specific tube based on its high-resolution AFM images, a discussion about the assignment of a cluster of chiral indices (n,m) by the numerical approximation method for each design, a discussion about the saddle-like model for tile oligomers to simultaneously grow "large" and "giant" tubes, additional AFM images of each assembly with descriptions, and sequence information. These materials are available free of charge via the Internet.

\section{AUTHOR INFORMATION}

\section{Corresponding Authors}

Nadrian C. Seeman - Department of Chemistry, New York University, New York, NY 10003, USA

https://orcid.org/0000-0002-9680-4649

Email : ned.seeman@nyu.edu

Shou-Jun Xiao - State Key Laboratory of Coordination Chemistry, School of Chemistry and Chemical Engineering, Nanjing University, Nanjing 210093, Jiangsu, China.

https://orcid.org/0000-0002-4240-1852

Email: sjxiao@nju.edu.cn

Author contributions: CJ, BL, and WZ contributed equally. SJX and NCS designed the research. CJ initiated and achieved LW-, bLW-, and TW-derived tiles and lattices, BL worked on the biotin/SA binding experiments, WZ worked on both lattice assembly and biotin/SA binding experiments. SJX, YPO, and NCS wrote the manuscript. BL, SJX, and CJ prepared the figures. FF and SL complemented the biotin/SA binding experiments for the bLW-E31/6 and DAE-E21/5 tubes, respectively. /All authors have given approval to the final version of the manuscript./

Notes

The authors declare no competing financial interest.

\section{ACKNOWLEDGMENT}

SJX acknowledges the financial support from the National Natural Science Foundation of China (Grant No. 91753134, 21571100). NCS acknowledges the financial support from Grant No. GM-29554 from NIGMS, Grant Nos. CTS1120890, CCF-1117210, EFRI-1332411, and CHE-1708776 from the NSF, Grant No. W911NF-1110024 from ARO, Grant Nos. N000141110729 and N000140911118 from ONR, DESC0007991 from DOE for partial salary support and GBMF3849 from the Gordon and Betty Moore Foundation.

\section{REFERENCES}

(1) Seeman, N. C. DNA in a material world. Nature 421, 427-431(2003).

(2) Winfree, E., Liu, F., Wenzler, L. A., Seeman, N. C. Design and self-assembly of two-dimensional DNA crystals. Nature 394, 539-544 (1998).

(3) Rothemund, P. W. K. Folding DNA to create nanoscale shapes and patterns. Nature 440, 297-302 (2006).

(4) Seeman, N. C., Sleiman, H. F. DNA nanotechnology. Nat. Rev. Mater. 3, 17068 (2017).

(5) Hong, F., Zhang, F., Liu, Y., Yan, H. DNA origami: scaffolds for creating higher order structures. Chem. Rev. 117, 12584-12640 (2017).

(6) Ramezani, H., Dietz, H., Building machines with DNA molecules. Nat. Rev. Genet. 21, 5-26 (2020).

(7) Aldaye, F. A., Palmer, A. L., Sleiman, H. F. Assembling materials with DNA as the guide. Science 321, 1795-1799 (2008).

(8) Hu, Q., Li, H., Wang, L., Gu, H., Fan, C. DNA nanotechnology-enabled drug delivery systems. Chem. Rev. 119, 6459-6506 (2019).

(9) Fu, T., Seeman, N. DNA double-crossover molecules. Biochemistry 32, 3211-3220 (1993).

(10) Liu, F., Sha, R., Seeman, N. C. Modifying the surface features of two-dimensional DNA crystals. J. Am. Chem. Soc. 121, 917-922 (1999).

(11) Mao, C., Sun, W., Seeman, N. C. Designed two-dimensional DNA Holliday junction arrays visualized by atomic forcemicroscopy. $J$. Am. Chem. Soc. 121, 5437-5443 (1999). 
(12) Zheng, J., Birktoft, J. J., Chen, Y., Wang, T., Sha, R., Constantinou, P. E., Ginell, S. L., Mao, C., Seeman, N. C. From molecular to macroscopic via the rational design of a self-assembled 3D DNA crystal. Nature, 461, 74-77 (2009).

(13) Liu, D., Wang, M., Deng, Z., Walulu, R., Mao, C. Tensegrity: construction of rigid DNA triangles with flexible four-arm DNA junctions. J. Am. Chem. Soc. 126, 2324-2325 (2004).

(14) Shih, W. M., Quispe, J. D., Joyce, G. F. A 1.7-kilobase single-stranded DNA that folds into a nanoscale octahedron. Nature 427, 618621 (2004).

(15) Zhang, C., He, Y., Chen, Y., Ribbe, A. E., Mao, C. Aligning one-dimensional DNA duplexes into two-dimensional crystals. J. Am. Chem. Soc., 129, 14134-14135 (2007).

(16) Yin, P., Hariadi, R. F., Sahu, S., Choi, H. M. T., Park, S. H., LaBean, T. H., Reif, J. H. Programming DNA tube circumferences. Science 321, 824-826 (2008).

(17) Ke, Y., Ong, L. L., Shih, W. M., Yin, P. Three-dimensional structures self-assembled from DNA bricks. Science 338, 1177-1183 (2012).

(18) Yan, H., Park, S. H., Finkelstein, G., Reif, J. H., LaBean, T. H. DNA-templated self-assembly of protein arrays and highly conductive nanowires. Science, 301, 1882-1884 (2003).

(19) Wang, P., Wu, S., Tian C., Yu, G., Jiang, W., Wang, G., Mao, C. Retrosynthetic analysis-guided breaking tile symmetry for the assembly of complex DNA nanostructures. J. Am. Chem. Soc. 138, 13579-13585 (2016).

(20) Dietz, H., Douglas, S. M., Shih, W. M. Folding DNA into twisted and curved nanoscale shapes. Science, 325, 725-730 (2009).

(21) Ke, Y., Douglas, S. M., Liu, M., Sharma, J., Cheng, A., Leung, A., Liu, Y., Shih, W. M., Yan, H. Multilayer DNA origami packed on a square lattice. J. Am. Chem. Soc. 131, 15903-15908 (2009).

(22) Woo, S., Rothemund, P. W. Programmable molecular recognition based on the geometry of DNA nanostructures. Nat. Chem., 3, 620627 (2011).

(23) Maier, A. M., Bae, W., Schiffels, D., Emmerig, J. F., Schiff, M., Liedl, T. Self-assembled DNA tubes forming helices of controlled diameter and chirality. ACS Nano 11, 1301-1306 (2017).

(24) Wei, B., Dai, M., Myhrvold, C., Ke, Y., Jungmann, R., Yin, P. Design space for complex DNA structures. J. Am. Chem. Soc. 135, 18080-18088 (2013).

(25) Marchi, A. N., Saaem, I., Vogen, B. N., Brown, S., LaBean, T. H. Toward larger DNA origami. Nano Lett. 14, 5740-5747 (2014).

(26) Benn, F., Haley, N. E. C., Lucas, A. E., Silvester, E., Helmi, S., Schreiber, R., Bath, J., Turberfield, A. J. Chiral DNA origami nanotubes with well-defined and addressable inside and outside surfaces. Angew. Chem. Int. Ed. 57, 7687-7690 (2018).

(27) Kim, Y.-J., Lee, C., Lee, J. G., Kim, D.-N. Configurational design of mechanical perturbation for fine control of twisted DNA origami structures. ACS Nano 13, 6348-6355 (2019).

(28) Mathieu, F., Liao, S., Kopatsch, J., Wang, T., Mao, C., Seeman, N. C. Six-helix bundles designed from DNA. Nano Lett. 5, 661-665 (2005).

(29) Hong, F., Jiang, S., Lan, X., Narayanan, R. P., Šlc, P., Zhang, F., Liu, Y., Yan, H. Layered-crossover tiles with precisely tunable angles for 2D and 3D DNA crystal engineering. J. Am. Chem. Soc. 140, 14670-14676 (2018).

(30) Ke, Y., Liu, Y., Zhang, J., Yan, H. A Study of DNA tube formation mechanisms using 4-, 8-, and 12-helix DNA nanostructures. J. Am. Chem. Soc. 128, 4414-4421 (2006).

(31) Mitchell, J. C., Harris, J. R., Malo, J., Bath, J., Turberfield, A. J. Self-assembly of chiral DNA nanotubes. J. Am. Chem. Soc. 126, 1634216343 (2004).

(32) Liu, X., Zhao, Y., Liu, P., Wang, L., Lin, J., Fan, C. Biomimetic DNA nanotubes: nanoscale channel design and applications. Angew. Chem. Int. Ed. Engl. 58, 8996-9011 (2019).

(33) Rothemund, P. W. K., Ekani-Nkodo, A., Papadakis, N., Kumar, A., Fygenson, D. K., Winfree, E. Design and characterization of programmable DNA nanotubes. J. Am. Chem. Soc. 126, 16344-16352 (2004).

(34) Green, L. N., Subramanian, H. K. K., Mardanlou,V., Kim, J., Hariadi, R. F., Franco, E. Autonomous dynamic control of DNA nanostructure self-assembly, Nat. Chem. 11, 510-520 (2019).

(35) Barish, R. D., Schulman, R., Rothemund, P. W. K., Winfree, E. An information-bearing seed for nucleating algorithmic self-assembly. Proc. Natl. Acad. Sci. U.S.A. 106, 6054-6059 (2009). 
(36) Mohammed, A. M., Schulman, R. Directing self-assembly of DNA nanotubes using programmable seeds. Nano Lett. 13, 4006-4013 (2013).

(37) Jorgenson, T. D., Mohammed, A. M., Agrawal, D. K., Schulman, R. Self-assembly of hierarchical DNA nanotube architectures with well-defined geometries. ACS Nano 11, 1927-1936 (2017).

(38) Sherman, W. B., Seeman, N. C. Design of minimally strained nucleic acid nanotubes. Biophys. J. 90, 4546-4557 (2006).

(39) Wang, T., Schiffels, D., Cuesta, S. M., Fygenson, D. K., Seeman, N. C. Design and characterization of 1D nanotubes and 2D periodic arrays self-assembled from DNA multi-helix bundles. J. Am. Chem. Soc. 134, 1606-1616 (2012).

(40) Woods, D., Doty, D., Myhrvold, C., Hui, J., Zhou, F., Yin, P., Winfree, E. Diverse and robust molecular algorithms using reprogrammable DNA self-assembly. Nature 567, 366-372 (2019).

(41) Guo, X., Wang, X.-M., Wei, S., Xiao, S.-J. Construction of a Holliday junction in small circular DNA molecules for stable motifs and two-dimensional lattices. ChemBioChem 19, 1379-1385 (2018).

(42) Guo, X., Wang, X. M., Xiao, S. J. Stable DNA motifs, 1D and 2D nanostructures constructed from small circular DNA molecules. J. Vis. Exp. 146, 58744 (2019).

(43) Dresselhaus, M. S., Dresselhaus, G., Saito, R. Physics of carbon nanotubes. Carbon 33, 883-891 (1995).

(44) Saito, R., Fujita, M., Dresselhaus, G., Dresselhaus, M. S. Electronic structure of chiral graphene tubules. Appl. Phys. Lett. 60, 22042206 (1992).

(45) Minev, D., Wintersinger, C. M., Ershova, A., Shih, W. M. Robust nucleation control via crisscross polymerization of highly coordinated DNA slats. Nat. Commun. 12, 1741 (2021).

(46) Reishus, D., Shaw, B., Brun, Y., Chelyapov, N., Adleman, L. Self-assembly of DNA double-double crossover complexes into highdensity, doubly connected, planar structures. J. Am. Chem. Soc. 127, 17590-17591 (2005).

(47) Lee, J. Y., Kim, Y.-J., Lee, C., Lee, J. G., Yagyu, H., Tabata, O., Kim, D.-N. Investigating the sequence-dependent mechanical properties of DNA nicks for applications in twisted DNA nanostructure design. Nucleic Acids Res. 47, 93-102 (2018).

(48) Jung, W. H., Chen, E., Veneziano, R., Gaitanaros, S., Chen, Y. Stretching DNA origami: Effect of nicks and Holliday junctions on the axial stiffness. Nucleic Acids Res. 48, 12407-12414 (2020). 